\title{
Corrigenda: Influence of self- compacting concrete on the lateral pressure on formwork
}

\author{
M. J. McCarthy, R. K. Dhir, S. Caliskan and M. K. Ashraf
}

Three typographical errors appeared in this paper when it was published in Structures and Buildings 165(3): 127-138, http://dx.doi.org/10.1680/ stbu.2012.165.3.127.

The second author's first name should have been given as Ravindra K. Dhir.

Two values in Table 3 were incorrect, the table with corrected values (emboldened) appears below.

\begin{tabular}{|c|c|c|c|c|c|c|c|}
\hline Reference & $\begin{array}{c}\text { Element } \\
\text { no. and } \\
\text { type }\end{array}$ & $\begin{array}{c}\text { Pour } \\
\text { height: } \\
\text { m* }\end{array}$ & $\begin{array}{l}\text { Additions } \\
\text { or fillers } \\
\text { in SCC }\end{array}$ & $\begin{array}{l}\text { Slump flow: } \\
\text { mm }\end{array}$ & $\begin{array}{c}\text { Concrete rate } \\
\text { of rise: } \mathrm{m} / \mathrm{h}\end{array}$ & $\begin{array}{c}\text { Maximum } \\
\text { pressure: } \\
\text { kN/m² }\end{array}$ & $\begin{array}{c}\text { Maximum } \\
\text { pressure/ } \\
\text { hydrostatic } \\
\text { pressure: \% }\end{array}$ \\
\hline \multirow[t]{3}{*}{ Bernabeu (2000) } & $8 W$ & $2 \cdot 1-7 \cdot 5$ & LS & $620-800$ & $6 \cdot 0-120 \cdot 0$ & $32 \cdot 5-111 \cdot 5$ & $29-100$ \\
\hline & $5 C$ & $2 \cdot 2-5 \cdot 0$ & LS & $580-890$ & $10 \cdot 0-150 \cdot 0$ & $46 \cdot 0-114 \cdot 5$ & $83-100$ \\
\hline & $3 C$ & $5 \cdot 0$ & LS & $720-890$ & $11 \cdot 0-60 \cdot 0$ & $110 \cdot 5-120 \cdot 5$ & $96-105$ \\
\hline Proske and Graubner (2002) & $5 C$ & $4 \cdot 0$ & FA & $550-750$ & $25-60$ & $40 \cdot 0-90 \cdot 0$ & $43-97$ \\
\hline Billberg (2003) & $7 W$ & $2 \cdot 9$ & LS & $650-780$ & $0 \cdot 8-2 \cdot 3$ & $12 \cdot 5-49 \cdot 5$ & $34-77$ \\
\hline \multirow[t]{2}{*}{ Leemann and Hoffmann (2003) } & $3 C$ & $2 \cdot 6$ & FA & $570-660$ & $8 \cdot 0$ & $50 \cdot 5-52 \cdot 0$ & $87-90$ \\
\hline & $1 \mathrm{~W}$ & $3 \cdot 9$ & $\mathrm{FA}^{\neq}$ & 700 & $18 \cdot 8$ & $97 \cdot 0$ & 110 \\
\hline \multirow[t]{2}{*}{ Vanhove et al. (2004) } & $1 C$ & $10 \cdot 4$ & LS & 700 & $10 \cdot 3$ & $175 \cdot 5$ & 74 \\
\hline & $1 \mathrm{C}$ & $10 \cdot 4$ & LS & 700 & $24 \cdot 0$ & $188 \cdot 5$ & 80 \\
\hline \multirow[t]{2}{*}{ Tejeda-Dominguez et al. (2005) } & $1 \mathrm{~W}$ & $7 \cdot 9$ & FA & $600-740$ & $1 \cdot 2$ & $38 \cdot 5$ & 24 \\
\hline & $1 C$ & $2 \cdot 9$ & FA & $600-740$ & $18 \cdot 3$ & $54 \cdot 5$ & 79 \\
\hline Assaad and Khayat (2006) & $2 \mathrm{C}$ & $2 \cdot 8$ & FAVSF & 540,745 & $10 \cdot 0$ & $49 \cdot 5,58 \cdot 0$ & 88,94 \\
\hline Cauberg and Desmyter (2007) & $3 C$ & $3 \cdot 8$ & NA & $685-810$ & $10 \cdot 0$ & $69 \cdot 0-85 \cdot 0$ & $85-97$ \\
\hline
\end{tabular}

* Height from top of element to lowest pressure measurement point; C: column; W: wall (using CIRIA 108 definition).

* Involved a retarder.

Shaded areas: Pumping from below, except one case (Bernabeu, 2000) when the discharge pipe was kept under SCC level during the pour. SF: silica fume; NA: information not available.

Table 3. Study details and comparison of maximum pressures with respect to hydrostatic pressures for SCCS (selective literature) 